\title{
Times of uncertainty
}

\author{
Epidemiological modelling informs government responses to the COVID-19 pandemic, but \\ confusion abounds about the models. What can physicists do to help?
}

\section{Cf even if every model parameter were well- quantified, most predictions won't come true}

During the COVID-19 pandemic, it has become common to see politicians give press conferences flanked by scientists and medical officers. Their presence provides a reassurance to the public that policy is 'led by the science', as the UK government has frequently put it. At the same time, questions have been raised by the press and in social media as to whether the modelling of the pandemic is valid. Media reports about models contain a variety of claims, the worst of which - such as a widely reported prediction of half a million deaths in the UK - appear not to be coming to pass. The range of predictions, and the apparent failure of models to describe reality, has led to negative reactions, and in extreme cases, a belief that the epidemic was never severe and business as usual should resume. But even for the physics community, which understands and appreciates modelling in general, the situation can be confusing.

One reason different modellers report different results is the scarcity of data to guide the choice of parameters, especially in the early stages of an epidemic. At first, epidemiologists can only estimate the parameters that concern the disease itself, such as how readily it is transmitted, and subsequently update the estimations. But such parameters describe the differences between seasonal influenza, COVID-19 and SARS or MERS. If they are wrong, the entire model must be revised. Furthermore, it is hard to come by real-time epidemiological data to fine-tune models against, because official data are often aggregated and reported with a delay, and small outbreaks may not be recorded at all. Indeed, a lot of research in the field of epidemiology is retrospective, looking at epidemics that have finished and for which better data exist, in order to learn lessons for the future.

But even if every model parameter were wellquantified, most predictions won't come true. Models don't say, 'this is what will happen' without prefacing it with 'if these assumptions are true, and in this particular scenario...' - they do not describe an unavoidable fate, but rather serve as a warning. There is also feedback at play. As communities respond to the pandemic, different models gain and lose relevance. For example, upon learning that inaction would lead to an extraordinary number of deaths, most governments chose to impose social distancing rules; thus, changes in policies led to changes in the models. In addition, policy-makers take advice not only from scientists, but also from economists, supply-chain experts, communicators and more - further complicating the feedback loop between models and reality.

In other words, epidemiological modelling is messy. It attempts to squeeze as much useful information as possible from limited noisy data that describe the complex nonlinear system of an infectious disease interacting with human society. There are no controlled experiments. Thus, although the equations that describe a susceptible-infectious-recovered epidemiological model are simple to write down, forecasting how a disease will spread, even in the short term, is a difficult business. In a Viewpoint in this Issue, mathematical epidemiologists comment further on the challenges in their work.

What can physicists do to help clear up confusion? As Julia Gog points out in a Worldview in this Issue, although the impulse to contribute to understanding the pandemic by constructing epidemic models may be strong, more useful ways to help include working with others to summarize the existing deluge of preprints and communicating the challenges of modelling to the public.

Such communication is vital because the scientific story of COVID-19 conflicts with the widespread view of science as a source of certainties. For the general public, if something is described by an equation, it is exact. Epidemiological modelling is a dramatic counter-example.

The contrast between the public conception of science and how it's actually practised is picked up in a second Viewpoint in this Issue, in which scientists involved in outreach discuss how to build public trust in science. A recurring theme is the need for scientists to talk about what they actually do, and not just their final results. We think the public confusion about modelling during this pandemic shows how true this is.

A call to arms to all physicists: with your mathematical training, you are in a strong position to understand the basics of how epidemiological modelling works. And for your non-scientist friends and family, you are an accessible and credible source of information. So next time you call your dad, explain the background to the media stories about models that he doesn't understand. 